\title{
PolytetraOuoroethylene patch saphenoplasty: Is it effective against recurrent varicose veins?
}

\author{
Hosam Roshdy, a MD; Khaled El Alfy, a MD; Tallal Amer,b MD
}

\author{
a) Department of General \& vascular Surgery, Faculty of Medicine, Mansoura \\ University, Egypt. \\ b)Department of Radiology, Faculty of Medicine, Mansoura University, Egypt.
}

Co"espondence:e-mail: hosam.roshdi@yahoo.com

\begin{abstract}
Background: Recurrent varicose veins after surgery (REVAS) are a common, complex and costly problem. The possibilities for recurrent varicose veins after correctly ligated SFJ may be due to: dilatation of preexisting venous tributaries from the common femoral vein $(C F V)$ or formation of new veins as a result of angiogenic stimulation termed neovascularisation. Interposition of a prosthetic implant covering the saphenous stump has been called "patch saphenoplasty." The aim of the present study is to evaluate the use of polytetrajluroethylene (PTFE) patch sutured over the saphenofemoraljunction (PTFE saphenoplasty) to reduce the incidence of recurrent varicose veins.

Patients and methods: This study was conducted in General and Vascular Surgery Unit at Mansoura University hospital between March 2007 and November 2010.Patients were included if they had varicose veins from the CEAP clinical classification: $C 2-5$; recurrent varicose veins and isolated short saphenous vein varicosity were excluded. 320 patients (368 limbs) were eligible for the study; The patients were randomized into two groups; group L flush ligation of the GSV was performed at the SFJ without using any technique to contain possible postoperative neovasculorization; group IL underwent PTFE saphenoplasty (rectangular piece $(2 \times 3 \mathrm{~cm})$ of PTFE was sutured on the saphenous stump).

Results: Both groups were comparable as regard patients' characteristics.The global incidence of early postoperative complications was higher in-group II than group I (9.4\% vs. 3.8\%) (PO.OOJ). Two months postoperative clinical and duplex examination revealed no clinical recurrence or neovascularization at the site of SFJ ligation in both groups.One-year postoperative clinical examination revealed recurrent thigh varicosities in 15/187 limbs in-group I (8\%) and in 8/181 limbs (4.4\%) in-group II. By duplex examination neovascularization occurred in $37 / 187$ (19.7\%) limbs in-group I (22 limbs grade 2 \& 15 limbs grade 3) but in-group II neovascularization occurred in 19/181(10.5\%) limbs (11 limbs grade 2 \& 8 limbs grade). (P 0.001$)$

Conclusion: Interposition of PTFE implant at the level of the ligated saphenofemoral stump significally reduces the incidences of clinical recurrence and neovascularization.

Key words: Polytetrajluoroethylene, saphenoplasty, varicose veins after surgery, neovascularization.
\end{abstract}

Introduction:

The saphenous venous system is the most commonly affected by varicose. Removal of diseased veins is still the standard surgical method. Great saphenous vein (GSV) stripping and ligation of the saphenofemoral junction
(SFJ) has been the most commonly performed operation for varicose veins (VV).1,2

Recurrent varicose veins after surgery (REVAS) are a common, complex and costly problem. The incidence lies between $20 \%$ and $80 \%$ depending on the definition of REVAS 
and the time to recurrence. 3 This extremely wide range of prevalence underscores the need for a better definition of this condition. An international consensus meeting held on REVAS in Paris (July 1998) agreed to adopt a clinical defmition of it, 4 i.e. the existence of varicose veins in a lower limb previously operated on for varicosities, with or without adjuvant therapies, which includes true recurrences, residual veins and new varicose, as a result of disease progression.

REVAS are a great problem for both patients and physicians. Operations for recurrence are more difficult and time consuming; complications are more common and can be serious. 4

Surgery in the form of saphenofemoral ligation (SFL), great saphenous vein (GSV) stripping and multiple phlebectomies remains the gold standard treatment for primary great saphenous varicose veins. Unfortunately, recurrence rates remain high, despite attempts to improve outcomes by ensuring that appropriately trained surgeons perform procedures.5

REVAS of the great saphenous vein (GSV) cannot always be attributed to technical inadequacy. Recent clinical studies have indicated that postoperative neovascularization may occur and can be detected on duplex ultrasound scans 6,7 Tiny new venous vessels developing in the granulation tissue around the saphenofemoraljunction (SFJ) may enlarge and connect to superficial veins, causing clinically obvious recurrence after a few years. 8,9

Surgical failure at the saphenofemoral junction- (SFJ) remains an important cause of recurrent varicose veins. It is generally agreed that recurrence is minimized by flush ligation of the great saphenous vein (GSV) at its junction with the femoral vein, together with ligation of all tributaries of the SFJ and any tributaries of the femoral vein in the region.6

Three potential mechanisms for reconnecting the deep and superficial venous systems after a correct SFJ ligation, with or without GSV stripping are; transnodal lymphovenous connections, enlargement of tiny femoral venules, and several variants of stump-related neovascularity.All of these occur on a background of the wound-healing process, in which angiogenesis is an important component, potentially giving rise to a more generalized, field-related neovascularity.10,11

The possibilities for recurrent varicose veins after correctly ligated SFJ may be due to: dilatation of preexisting venous tributaries from the common femoral vein (CFV); 9,12 or formation of new veins because of angiogenic stimulation termed neovascularisation (NV). Many authors favour the neovascularisation4,13.

It seems that apart from correctly performed preoperative diagnosis and adequate surgery (including flush saphenous ligation), other measures are necessary in order to avoid recurrence. These measures should be directed towards prevention or at least limitation of the extent of neovascularisation. 14

Multiple surgical techniques were used aiming to create a physical barrier between the ligated stump on the common femoral vein (CFV) and the surrounding superficial veins. Over the years, several techniques have been tested with varying results. Closure of the cribriform fascia and covering the saphenous stump with an artificial implant was apparently associated - with good results at clinical follow up.6,7

Interposition of a prosthetic implant covering the saphenous stump is called "patch saphenoplasty". Various patch materials have been used for this purpose: Mersilene mesh, reinforced silicone sheeting, polytetrafluoroethylene (PTFE), and Dacron. ${ }^{15},{ }^{1} 6$

The aim of the present study is to evaluate the use of polytetrafluro ethylene (PTFE) patch sutured over the saphenofemoral junction (PTFE saphenoplasty) to reduce the incidence of recurrent varicose veins.

\section{Patients and methods:}

This study was conducted in General and Vascular Surgery Unit at Mansoura University hospital between March 2007 and November 2010

Patients were included if they had varicose veins from the CEAP clinical classification: C2-5.

Recurrent varicose veins, isolated short 
saphenous vein varicosity and active ulceration (C6) were excluded.

320 patients (368 limbs) were eligible for the study; informed written consent was obtained from all patients.

The patients were thenrandomized into two groups:Group(I), flush ligation of the GSV was performed at the SFJ without using any technique to contain possible postoperative neovasculorization; group(II) underwentPTFE saphenoplasty.

Randomization was performed using sealed envelopes and the random number generator with varying block sizes in SPSS. Envelopes were opened on the day of surgery, patients rather than individual legs were randomized; thereforet those with bilateral varicose veins had the same procedure performed on both legs.

All patients underwent assessment preoperatively with clinical examination and duplex imaging.

A single surgical team performed all operations.

All operation were performed under spinal anesthesia (safe to the patients and avoid complication of general anesthesia) except 17 patients done under general anesthesia either due to failure of spinal anesthesia or the patients refused spinal anesthesia.

Surgical procedures:

Group(I): After incision in the skin crease of the groint the GSV was exposed and all tributaries divided between ligatures. When

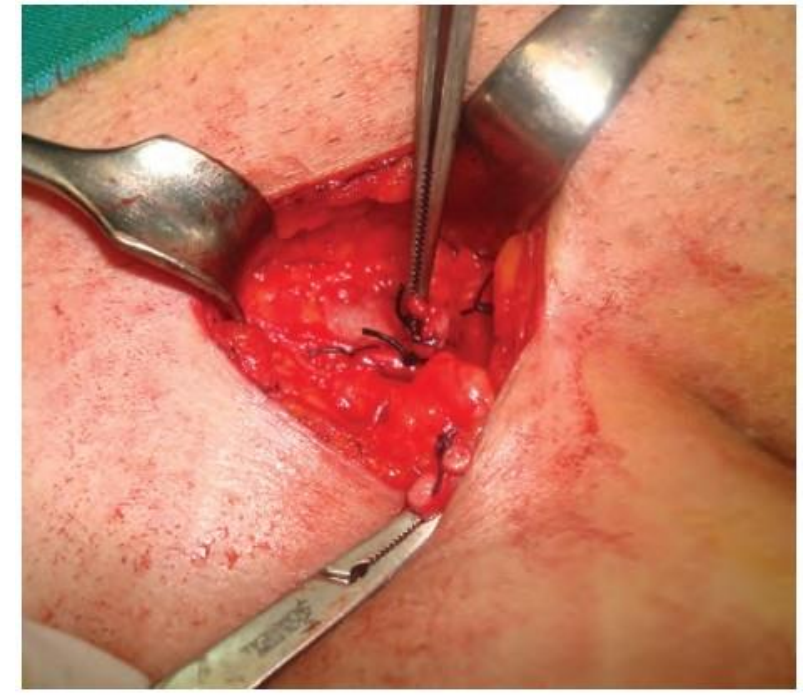

(A) Flush ligation at saphenofemoral junction, with ligation of all tributaries the termination of the GSV in the common femoral vein bad been identified with ce.rfllinty, the main trunk of the GSV was divided and the SFJ was exposed through the opening in the cribriform fascia, without enlarging it. Tributaries ending - directly into the common femoral vein within $1 \mathrm{em}$ above or below the SFJ were separately ligated Flush ligation of the GSV was performed at the SFJ with Silk $2 / 0$ followed by stripping of the GSV to the level of the knee and stab avulsion phlebectomies of established varicose clusters.

The groin incision was closed with redivac drain without using any technique to contain possible postoperative neovasculoriza.tion.17,18

Group(II): The same operation was performed as a part from the procedure in the groin but before closing the groin incision, a rectangular piece $(2 \times 3 \mathrm{~cm})$ of PTFE was sutured on the saphenous stump. The PTFE implant was tucked under the cribriform fascia, in order to cover the anterior half of the deep vein in the neighborhood of the saphenous stump. The opening in the cribriform fascia was closed by vicryl $3 / 0$ in separate stitches to maintain the patch inapposition to the deep vein.

Our usual practice when treating patients with bilateral varicose veins is to treat one limb at a time, leaving a period of three months between the first and the second operation. In this way, we allow complete recovery of the patient and resolution of symptoms and signs following surgical treatment.

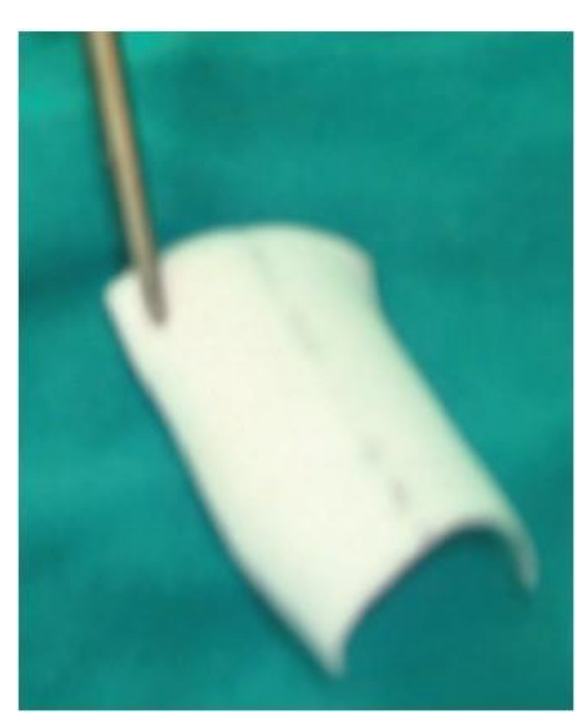

(B) Polytetrajlouroethylene patch 


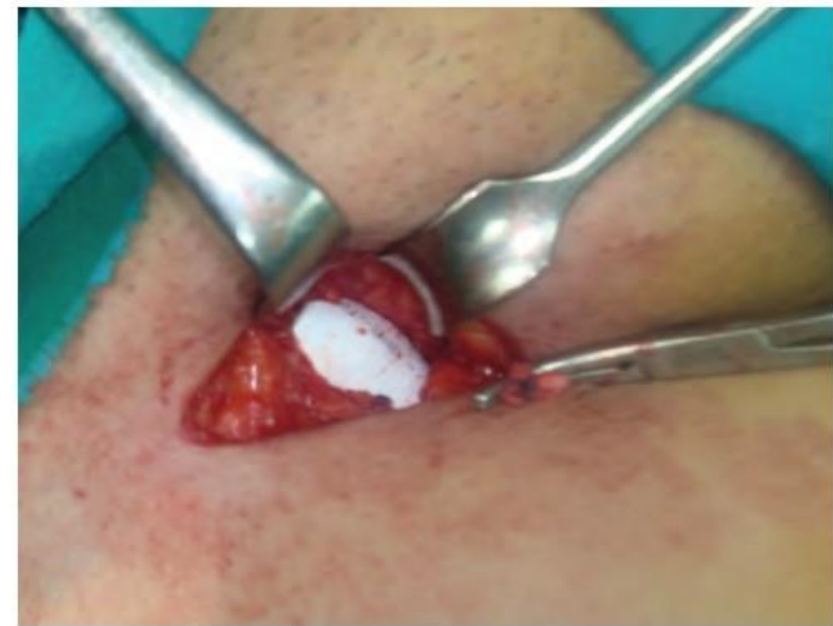

(C) Fixation ofPTFE patch at saphenofemoral ligation on the femoral vein

Follow up:

Follow up period range from 6 to 36 months (median 21 months) post operation; all patients underwent postoperative clinical and duplex examination 2 and 12 months postoperatively (after 12 months 23 patients (27limbs) were lost to follow up).

At clinical examination patients were checked for the presence of recurrent varicose veins i.e.recurrent varicose veins were defined as any new visible varicosity on clinical examination one year post operatively (palpable, dilated subcutaneous veins larger than $4 \mathrm{~mm}$ ).

Post operative duplex scanning for the presence or absence of neovascularization.

Neovascularisation was defined as the presence of serpentine venous tributaries entering the common femoral vein at the site of the old saphenofemoral junction after calf compression or Valsalva's maneuver.A clinical grading system was used to describe the degree of neovascularisation. 19,20

Grade 0: no neovascularisation

Gradel: $<3 \mathrm{~mm}$ diameter vessels

Grade 2: $>3 \mathrm{~mm}$ diameter vessels with visible reflux.

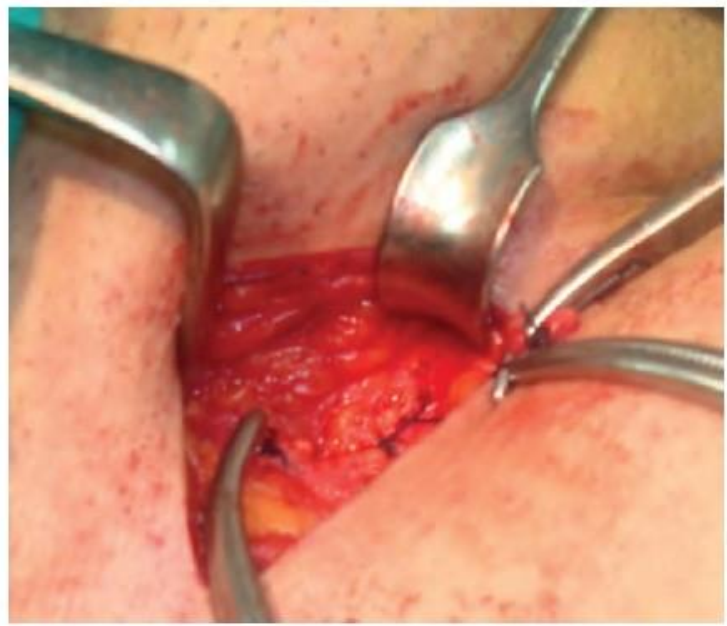

(D) Closure of cribriform fascia over the patch

Statistical analysis:

The statistical analysis of data was done by using excel program and SPSS program statistical package for social science version 16.

The description of the data was done in the form of mean(+/-) SD for quantitative data; and Frequency \& proportion for Qualitative data.

The analysis of the data was done to test statistical significant difference between groups. For quantitative data, independent sample ttest was used to compare between two groups.

Chi square test was used for qualitative data.

N.B:P is significant if $<$ or $=0.05$ at confidence interval $95 \%$.

\section{Results:}

Three hundreds and twenty patients (368 limbs) were included in the study. Their mean ages in-groupi and Groupll were $(40 \pm 18$.) and $(39 \pm 18.7)$ years respectively. There is no significant difference regarding age and sex between the two groups. Both groups were comparable regarding patients' characteristics Table(1). 
Table (1): Patients' characteristics.

\begin{tabular}{|l|c|c|}
\hline & Group I & Group II \\
\hline Number of patient & 160 & 160 \\
Number of legs & 187 & 181 \\
Mean age (range) & $40(19-62)$ & $39(20-61)$ \\
Unilateral & 133 patients & 139 \\
Bilateral $\quad$ Male & 27 & 21 \\
Sex: Female & 65 & 61 \\
& 95 & 99 \\
\hline
\end{tabular}

There is no significant difference as regard clinical classification of the

patients by CEAP classification Table (2).

Table (2): C of CEAP classification.

\begin{tabular}{|c|c|c|}
\hline & Group I & Groupll \\
\hline $\mathrm{C} 2$ & 35 & 31 \\
\hline $\mathrm{C} 3$ & 74 & 85 \\
\hline $\mathrm{C} 4$ & 52 & 47 \\
\hline C5 & 26 & 18 \\
\hline Total & 187 legs & 181 legs \\
\hline
\end{tabular}

The global incidence of early postoperative complication was higher in-group IT than group I (9.4\% vs. $3.8 \%$ ) (P0.001) Table(3).

Wound infection occurred in six limbs ingroup IT and in two limbs in-group I all resolved by antibiotics except in one limb that hadPTFE patch in which the patch had to be removed.

Lymphatic problems in the form of lymphocele \& lymphoedema occurred in ten limbs (8 Lymphocele \& 2 lymphoedema) ingroup II. However, in-group I lymphatic problems occurred in four limbs (2lymphocele
\& 2 lymphoedema). Lymphocele disappeared after evacuation of the lymphatic fluid lymphoedema, which was transient and resolved during follow up.

Asymptomatic deep venous thrombosis (DVT), at the level of common femoral vein in 2 patients (one in group and one in group II) which was discovered by Duplex scan 2 months after the operation on these patients was treated by oral anticoagulants for 6 months and resulted in complete recanalization of the vern.

\section{Table (3): Complication.}

\begin{tabular}{|l|c|c|}
\hline & Group I & Group II \\
\hline Wound infection & 2limbs & $61 \mathrm{imbs}$ \\
Lymphatic problems & 4 limbs & 10 limbs \\
-lymphocele & 2 & 8 \\
-lymphaedema & 1 & 2 \\
PartialDVT & 1 & 1 \\
\hline
\end{tabular}


- Two months postoperative clinical and duplex examination revealed no clinical recurrence or neovascularization at the site of SFJ ligation in both groups.

-After one year clinical examination revealed recurrent thigh varicosities in 15 limbs in group I (8\%) and in 8 limbs $(4.4 \%)$ in group II. And by duplex examination neovascularization occurred in $37 / 187(19.7 \%)$ limbs in group I (22 limbs grade $2 \& 15$ limbs grade 3) but in group II neovascularization occurred in 19/181(10.5\%) limbs (11 limbs grade 2 \& 8 limbs grade).

(P 0.001)

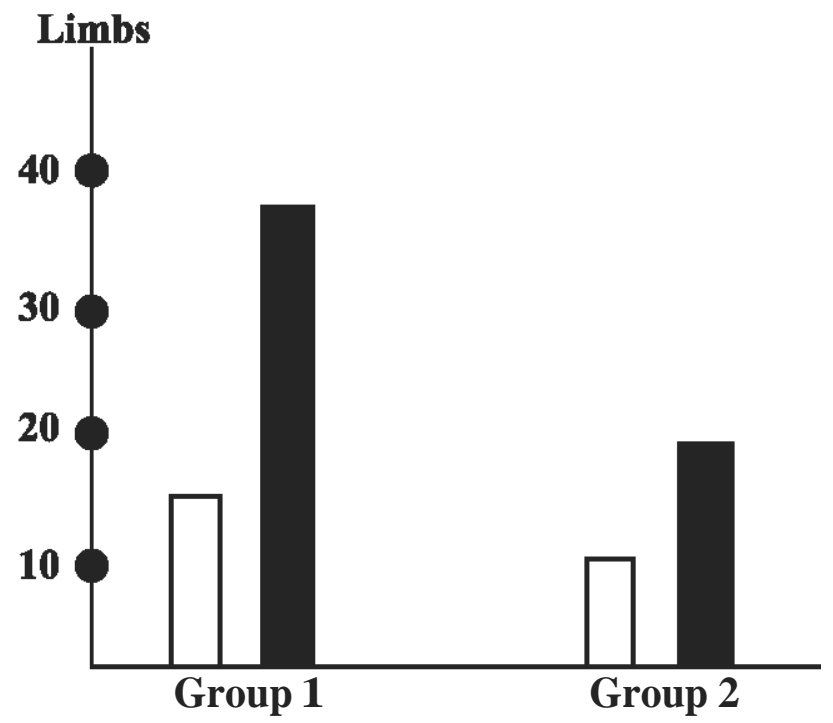

Figure (2): Total incidence of clinical recurrence and neovascularization by in both groups one year Postoperatively.

\section{Discussion:}

The management of venous disease of the lower extremities was greatly improved with the advent of the hand - held Doppler and was again substantially enhanced by color-coded duplex imaging_1O

The surgical treatment of varicose veins has changed very little over the last century. Many studies have explored the importance of stripping the GSV with a wide range of reported recurrence rates $(7-65 \%) .21$

The surgical treatment of primary varicose veins associated with GSV reflux should include flush SFJ ligationt excision of all tributaries entering the terminal portion of the GSVt stripping the thigh portion of the GSV, and stab avulsion phlebotomies of established varicose clusters.ll

Inappropriate use of the term recurrence might partly explain the considerable variation between different studies. VV after a previous operation may be residual varicose, true recurrences or progression of the disease - in new vein segments. 22

There are - three major sources of recurrence following varicose vein surgery. The first group of causes is attributable to inadequate or incomplete initial treatment. These arise either due to a tactical error resulting in failure to identify all incompetent veins or due to failure to carry out technically adequate primary treatment (despite a correct preoperative diagnosis). The second group of causes arises from the progression of venous disease resulting in development of varicose in previously normal veins. The third cause of recurrence is neovascularisation where varicose arise in the track of previously stripped or ligated veins. ${ }^{2} 3$

Recurrent varicose veins after ligation of the SFJ can be divided in those caused by technical inadequacy or those without operative error. $24.2^{5}$ Botht in our study and in the studies of Creton 15 and De Maeseneer17 operative mistakes could be excluded by early postoperative duplex imaging. When the first operation had been performed correctly, there are two potential pathogenic mechanisms for new reflux have been postulated.Dilatation of pre-existing veins or true angiogenesis. Firstlyt dilatation of pre-existing veins may occur in venules in a lymph node venous network, or 
it may follow dilatation of small adventitial vessels in the vasa vasorum of the femoral vein.25 Alternatively, it may be the result of collaterals. Any of these dilated - tributaries may create a new connection between the femoral vein and any residual superficial veins left in the thigh. The second pathogenic mechanism neovascularisation-might be stimulated by the free endothelium left after simple saphenofemoral ligation by vascularisation of residual thrombus or by disturbed venous drainage of the ligated branches of the SFJ.9,26

The fact that there are recurrent $\mathrm{VV}$ does not mean that the patient suffers from any discomfort, i.e. the recurrent VV may be without clinical significance; this also makes figures about recurrence rates difficult to interpret. Nevertheless, re-operations for VV are common, and the increased knowledge from various duplex-based studies on the anatomy of $\mathrm{VV}$ and patterns of recurrences has so far not improved the operative results.27

A significant amount of research has been aimed at decreasing recurrent groin reflux. Barrier techniques have been used by a number - of authors. Sheppard was the first to describe the use of an anatomical barrier, in the form of a reflected flap of pectineus fascia. 28

Gibbs used a reflected flap of pectineus fascia and showed no difference in rerecurrence rates inpatients with recurrent varicose veins. 29

Glass pioneered the use of a synthetic barrier in the form of a synthetic mesh (Mersilene, Ethicon Ltd). He showed a SFJ recurrence rate of $1 \%$ at a mean of 5 years following surgery. 6

A number of different synthetic materials have been used including silicone patches and polytetrafluoroethylene (PTFE) patches. De Maeseneer showed lower rates of neovascularisation in patients who had a silicone patch inserted over the ligated SFJ.16,18

Creton between April1992 and June 1994 first used PTFE. Patients with recurrent varicose veins underwent redo saphenofemoralligation followed by insertion of a PTFE patch ( 1 x 1.5 em), which was glued, to the CFV. Follow up at a mean of 4.9 years showed a neovascularisation rate of $4 \% .15$

In our study, we have used PTFE patches.
The preliminary results ofPTFE patch saphenoplasty reported by Earnshaw were less promising than the results of the present study. In $14(21 \%)$ of 66 limbs- (51 primary VV and 15 recurrences) operated upon with this barrier technique recurrent VV were visible after one year. On duplex scan neovascularisation at the SFJ ligation site accounted for 10 of 14 recurrences. Although PTFE is an ideal impervious material, the design of the quite small PTFE patch $(1 \times 2$ em) seemed to be unsatisfying and therefore the size of the patch was increased to $3 \times 2$ cm. 6,7

A pilot study done by Jones et al, involving patients with primary and recurrent veins showed that use of the patch was safe. Those undergoing surgery for recurrence had disappointing results with a recurrence rate of $47 \%$ (7 of 15 legs) and neovascularisation rate of $40 \%$ (6 of 15legs). A further 80 legs with recurrence veins underwent patch insertion and the recurrence rates and neovascularisation rates at a median of 19 months following surgery were $23 \%$ and $37 \%$ respectively. Neovascularisation remained the commonest source of recurrence.19

In our study PTFE patch was tailored to 2 x $3 \mathrm{em}$ in order to cover the anterior half of the common femoral vein. The PTFE patch was tucked underneath the cribriform fascia then covering the patch by suturing the cribriform fascia over it creating a double barrier, which may be an explanation for our improved results.

In our study, neovascularisation occurred in $(19.7 \%)$ in group I and $(10.5 \%)$ in group II. These results were compared with a previously reported study using silicone patch saphenoplasty and another group without any barrier technique. The incidence of neovascularisation at one year follow-up in the anatomical barrier group (6.7\%) was significantly lower than in the group without barrier (14.8\%; P 0.001) similar to the incidence reported after patch saphenoplasty (5.2\%: $\mathrm{P}=0.45) \quad(17,18)$.

As regard complication De maesseneer et al.,16 reported the incidence of complications after silicone patch saphenoplasty in which wound infection occurred in $2 \%$ lymphocele 
or lymyhoedema in $3.2 \%$, asymptomatic venous thromboembolism in $1 \%$ \& these results were nearly equal to the results in our study and nearly equal to that reported by Winterbom et al.JO

\section{Conclusion:}

Interposition of PTFE implant at the level of the ligated saphinofemoral stump significally reduces the incidence of clinical recurrence and neovascularization.

\section{References:}

1- Caggiati A, Bergan J, Gloviczki P, Jantet G, Wendell CP, Partsch H: Nomenclature of the veins of the lower limbs: An international interdisciplinary consensus statement. Vase Surg 2002; 36(2): 416422.

2- Rucldey CV, Bradburg AW: How do we prevent recurrence of varicose veins? In: Venous disease. Epidemiology, management and delivery of care. Rucldey CV, Fowkes FGR, Bradbury AW (Editors); London: Springer (Publisher); 1999; p. 239-245.

3- Glass GM: Prevention of recurrent saphenofemoral incompetence through neovascularization after surgery for varicose veins. BrJ Surg 1999; 76:1210.

4- Perrin MR, Guex JJ, Rucldey V, De Palma RG, Royle JP, Eklof B, et al: Recurrent varices after surgery (REVAS), a consensus document. Cardiovase Surg 2000; 8: 233 245.

5- De Maeseneer M, Philipsen T, Vandenbroeck C, Lauwers P, Hendriks J, Hert S, Van Schil P: Closure of the cribriform fascia: An effective anatomical barrier against postoperative neovascularisation at the saphenofemoral junction: A Prospective Study. Eur J Vase Endovasc Surg 2007; 34: 361-366.

6- Glass GM: Prevention of saphcnofemoral and saphenopopliteal recurrence of varicose veins by forming a partition to contain neovascularization. Phlebology 1998; 13 : 3-9.

7- Earnshaw JJ, Davies B, Harradine K, Heather BP: Preliminary results of PTFE patch saphenoplasty to prevent neovascularization leading to recurrent varicose veins. Phlebology 1998; 13: 1013.

8- van Rij AM, Jones GT, Hill GB, Hons BS, Jiang P:Neovascularization and recurrent varicose veins: More histologic and ultrasound evidence. J Vase Surg 2004; 40: 296-302.

9- Fischer R, Chandler JG, De Maescnccr MG, et al: The unresolved problem of recurrent saphenofemoral reflux. JAm Coli Surg 2002; 195: 80-94.

10-Dwerryhouse S, Davies B, Harradine K, Earnshaw JJ:Stripping the long saphenous vein reduces the rate of reoperation for recurrent varicose veins. JVase Surg 1999; 29: 589-592.

11-Fischer R, LindeN, Duff C, et al: Late recurrent saphenofemoral junction reflux after ligation and stripping of the greater saphenous vein.J Vasc Surg 2001; 34: 236240.

12-Frings N, Nelle A, Tran P,Glowacki P: Unavoidable recurrence and neoreflux after correctly performed ligation of the saphenofemoral junction: Neovascularisation? Phlebology 2003; 32: 96-100.

13-Viani MP, Poggi RV, Pinto A, et al: Reexploration of the saphenofemoral junction in the treatment of recurrent varicose veins. Int Surg 1996; 81: 382-384.

14-Coleridge S P: Prevention of recurrence of varicose veins following surgery (editorial). Phlebology 1998; 13: 1-2.

15-Creton D: Surgery for recurrent saphenafemoral incompetence using expanded polytetrafluoroethylene patch interposition in front of the femoral vein: Long term outcome in 119 extremities. Phlebology 2002; 16: 93-97.

16-De Maeseneer MG, Vandenbroeck CP, Van Schil PE: Silicone patch saphenoplasty to prevent repeat recurrence after surgery to treat recurrent saphenofemoral incompetence: Long-term follow-up study. JVase Surg 2004; 40(1).

17-De Maeseneer MG, Tielliu IF, Van Schil PE, De Heit SG, Eyskens EJ: Clinical relevance of neovascularisation on duplex ultrasound in the long term follow up after 
varicose vein operation. Phlebology 1999; 14: 118-122.

18-De Maeseneer MG, Giuuani DR, Van Schil PE, De Hert SG: Can interposition of a silicone implant after sapheno-femoral ligation prevent recurrent varicose veins? Eur JVase Endovase Sure 2002; 24: 445449.

19-Jones L, Braithwaite BD, Selwyn D, Cooke S, Earnshaw JJ: Neovascularisation is the principal cause of varicose vein recurrence: Results of a randomised trial of stripping the long saphenous-vein. Eur J Vase Endovase Surg 1996; 12: 442-445.

20-Nyamekye I, Shepard NA, Davies B, Heather BP, Earnshaw JJ: Clinicopathological evidence that neovascularisation is a cause of recurrent varicose veins. Eur JVase Endovase Surg 1998; 15: 412-415.

21-WinterbomRJ, FoyC, Earnshaw JJ:Causes of varicose vein recurrence: Late results of a randomized controlled trial of stripping the long saphenous vein.JVase Surg 2004; 40: 634-639.

22-Blomgren L, Johansson G, Akerman A, Noren A, Brundin C, Nordstrom E, Bergqvist D: Recurrent varicose veins: Incidence, risk factors and groin anatomy. Eur JVase Endovase Surg 2004; 27: 269274.

23-Kostas T, Ioannou CV, Touloupakes E, Dastalaki E, Giuannoukas AD, Tsetis D, et al: Recurrent varicose veins after surgery: A new appraisal of a common and complex problem in vascular surgery. Eur J Vase Endovase Surg 2004; 27: 275-282.

24-Fischer R, Kluess HG, Frings N, Duff C: Present state of saphenofemoral recurrence research. Phlebology 2003; 32: 54-59.

25-Kohler A, Dirsch O, Brunner U: Venolymphatic angiodysplasia as cause of an inguinal recurrent varicosis. Vasa 1997; 26: 52-54.

26-Frings N, Tran V A, Glowacki P: Recurrence of saphenofemoral junction despite correctly performed ligation of SFJ. Phlebology 1999; 28: 144-148.

27-Allergra C, Antignani P, Carlizza A: Recurrent varicose veins following surgical treatment: Our experience with five years follow up. Eur JVase Endovase Surg 2007; 33: 751-756.

28-Sheppard M:A procedure for the prevention of recurrent saphenofemoral incompetence. Aust NZ J Surg 1978; 48: 322-326.

29-Gibbs PJ, Foy DM, Darke SG: Reoperation for recurrent saphcnofemoral incompetence: A prospective randomized trial using a reflected flap of pectineus fascia. Eur $J$ Vas Endovas Surg 1999; 18: 494-498.

30-Winterbom RJ, Foy C,Heather B P, Earnshaw J J: Randomised trial of flush saphenofempralligation for primary great saphenous varicose veins. Eur J Vase Endovase Surg 2008; 36: 477-484. 